\title{
Discapacidad y alojamientos turísticos en España
}

\author{
Trinidad Domínguez Vila* \\ J. Antonio Fraiz Brea** \\ $M^{a}$ Elisa Alén González***
}

\author{
Universidade de Vigo (España)
}

\begin{abstract}
Resumen: El turismo accesible representa un segmento con gran potencialidad y generador de una ventaja competitiva para el sector, dado que los beneficiarios de acciones de accesibilidad engloban a colectivos en crecimiento como el de las personas mayores, las familias, personas con capacidades restringidas temporalmente y embarazadas. Su representatividad en el total de la actividad turística española está en incremento continuo, motivado por dos factores principales: la benevolencia climática y la normativa y cobertura social existente. Por todo ello, en esta investigación se busca determinar los principales hábitos y comportamientos de los turistas españoles con discapacidad en relación al alojamiento utilizado en sus viajes, así como determinar cuáles son las variables que pueden influir en dicho comportamiento.
\end{abstract}

Palabras Clave: turismo accesible, discapacidad, alojamiento, hábitos y comportamiento.

\section{Disability and touristic accommodation in Spain}

Abstract: Accessible tourism represents a segment with high potential and generator of a competitive advantage for the tourism sector. This profit is a result of all people beneficiary of accessibility as elderly people, who is increasing, families, people with temporary disabilities and pregnant women. Their representatively over the total of the Spanish tourism industry is growing because of two main factors: benevolence climate and regulations and social security coverage. Therefore, this research seeks to identify the key habits and behavior of Spanish tourist with disabilities about the accommodation that they use in their trips, and the main variables which can influence in their behavior.

Keywords: accessible tourism, disability, accommodation, behavior and habits.

\section{Introducción}

El interés que se ha generado en los últimos años a cerca del turismo para personas con discapacidad o turismo accesible es notorio, reflejo de ello es el incremento de las investigaciones vinculadas al turismo y la discapacidad, donde destacan las enfocadas a las barreras con las que se encuentra dicho segmento (Daniels, Rodgers, y Wiggins, 2005; Nyaupane y Andereck, 2008; Turco, Stumbo, y Garncarz, 1998), las relacionadas con el mercado (Burnett y Bender-Baker, 2001; Domínguez, Fraiz, y Alén, 2013; Dwyer y Darcy, 2011; Van Horn, 2012), las que trabajan temas de motivación (Figueiredo, Eusébio, y Kastenholz, 2012; Ray y Ryder, 2003; Shi, Cole, y Chancellor, 2012), las que se centran en las necesidades de información (Buhalis y Michopouloub, 2011; Darcy, 2010; Eichhorn, Miller, Michopoulou, y Buhalis, 2008), o en el comportamiento y actitudes de los proveedores (Darcy y Pegg, 2011; Gröschl, 2007, 2012; Kim, Stonesifer, y Han, 2012; McKercher, Packer, Yau, y Lam, 2003; O’Neill y Ali Knight, 2000; Ozturk, Yayli, y Yesiltas, 2008; Patterson, Darcy, y Monninghoff, 2012; Tantawy, Kim, y Pyo, 2005; Yaniv, Arie, y Yael, 2011), entre otras.

\footnotetext{
* E-mail: trinidad@uvigo.es

** E-mail:jafraiz@uvigo.es

*** E-mail: alen@uvigo.es
} 
Aunque cada vez existen más investigaciones sobre personas con discapacidad y su tiempo de ocio, muchas de sus necesidades en términos turísticos aún son ignoradas o poco estudiadas por la academia (Ray y Ryder, 2003, Burnett y Baker, 2001), sin existir un verdadero esfuerzo por parte de los investigadores y responsables de turismo (Daniels et al., 2004).

En general, el turismo para personas con discapacidad o turismo accesible, hace referencia al proceso requerido para asegurar que el transporte, alojamiento, destinos y atracciones de todo el sistema turístico cumplen adecuadamente las necesidades de las personas con discapacidad, así como a otros colectivos que se pueden beneficiar de mejoras a nivel de movilidad, visión, audición o cognitivamente (Buhalis y Darcy, 2011). A nivel mundial se estima que entre el 9\% y el 13\% de la población padece alguna discapacidad (Horgan-Jones y Ringaert, 2004; Van Horn, 2002), lo que representa aproximadamente a 650 millones de personas (Naciones Unidas, 2006). Para 2050, se prevé que dicha estimación se incremente hasta los 1.200 millones de personas (Naciones Unidas y Banco Mundial, 2011).

El trabajo que se presenta a continuación, pretende mostrar la importancia del turismo accesible a través de su vinculación al sector turístico en base a las demandas y los potenciales beneficios económicos que pueden generar. En base a ello, su buscará mostrar las oportunidades de mercado que se generan con dicho segmento y mostrar sus hábitos y comportamientos a la hora de viajar, especialmente centrado en el alojamiento. Se toma como unidad de estudio a los turistas españoles con discapacidad, esta elección está estrechamente ligada a la gran tradición turística y a la importancia que representa dicho sector para la economía española. Según datos de la Organización Mundial de Turismo -OMT- (2013), España es el cuarto destino mundial en recepción de turistas (57,7 millones) y el segundo destino mundial de ingresos por turismo con 55.900 millones de dólares, donde más del $15 \%$ de empresas y el $20 \%$ de los trabajadores están relacionados con el sector turístico. Por lo que queda claro, que el sector debe centralizar sus políticas y estrategias en nuevos segmentos, como son los turistas con discapacidad y turistas senior, caracterizados por la búsqueda de calidad y con alto grado de lealtad al destino.

\section{El turismo accesible y su potencialidad}

Cuando se habla de datos en relación a las personas con discapacidad, las cifras pueden variar mucho según la fuente que se cite (ver Tabla 1). Este hecho viene dado por la variación de definiciones y coberturas aplicadas al término discapacidad.

Tabla 1: Personas con discapacidad. Demanda general

\begin{tabular}{|c|c|c|c|}
\hline \multicolumn{4}{|c|}{ DEMANDA POTENCIAL O GENERAL } \\
\hline Lugar & Estimación & $\begin{array}{c}\text { \% de la } \\
\text { población }\end{array}$ & Fuente o referencia \\
\hline Mundo & De 600 a 859 millones de personas. & Del $9 \%$ al $13 \%$ & $\begin{array}{l}\text { - Van Horn, } 2002 . \\
\text { - Horgan - Jones y Ringaert, } \\
2004 .\end{array}$ \\
\hline USA & Cerca de 54 millones de personas. & $21 \%$ & $\begin{array}{l}\text { - U.S. Departament of } \\
\text { Commerce, } 1997 .\end{array}$ \\
\hline Australia & De 50 a 80 millones de personas. & Del $16 \%$ al $26 \%$ & - Stumbo y Pegg, 2005. \\
\hline \multirow{3}{*}{ Europa } & $\begin{array}{l}\text { Más de } 3 \text { millones de personas en } \\
1993 .\end{array}$ & $18 \%$ & - Darcy, 1998. \\
\hline & $\begin{array}{l}\text { Sobre } 45 \text { millones en la UE-25 } \\
\text { países (edades de } 16 \text { a } 64 \text { años) }\end{array}$ & $15,7 \%$ & $\begin{array}{l}\text { - Dupré y Karjalainen, } \\
\text { publicado Eurostat (2003). }\end{array}$ \\
\hline & $\begin{array}{l}\text { De } 45 \text { a } 90 \text { millones que tengan } \\
\text { algún tipo de impedimento. }\end{array}$ & Del $10 \%$ al $20 \%$ & $\begin{array}{l}\text { - Teorisme Vlaanderen (2001). } \\
\text { - Nacional Disability } \\
\text { Authority (2003). }\end{array}$ \\
\hline
\end{tabular}




\begin{tabular}{|c|c|c|c|}
\hline \multicolumn{4}{|c|}{ DEMANDA POTENCIAL O GENERAL } \\
\hline \multirow[t]{4}{*}{ Europa } & 50 millones en la Europa ampliada. & Aprox. $11 \%$ & $\begin{aligned} & \text { - European Disability Forum, } \text { } \\
& 2005 . \\
& \text { - Gerlin, } 2005 . \\
& \text { - Qualitas, } 2004 . \\
& \text { - Brown, } 1991 . \\
& \text { - Van Horn, } 2002 . \\
& \text { - Horgan-Jones y Ringaert, } \\
& \\
& \quad \text { 2004. }\end{aligned}$ \\
\hline & De 69 a 92 millones de personas. & Del $15 \%$ al $20 \%$ & - Pühretmair, 2004. \\
\hline & $\begin{array}{l}\text { De } 60 \text { a } 80 \text { millones de personas } \\
\text { discapacitadas y con movilidad } \\
\text { reducida. }\end{array}$ & Del $13 \%$ al $17 \%$ & $\begin{array}{l}\text { - Community Research and } \\
\text { Development Information } \\
\text { Service CORDIS, } 1995 .\end{array}$ \\
\hline & De 92 a 115 millones de personas. & Del $20 \%$ al $25 \%$ & - Stumbo y Pegg, 2005. \\
\hline
\end{tabular}

Si se realiza un estudio más profundo sobre el segmento de personas beneficiarias de la supresión de barreras en España (ver Tabla 2), la cifra se sitúa alrededor de los 16 millones de personas, lo que constituye casi el 40\% de la población española (Ministerio de Trabajo y Asuntos Sociales, 2003:28).

Tabla 2: Personas beneficiarias de la supresión de barreas en España

\begin{tabular}{|c|c|c|c|}
\hline Beneficiarios accesibilidad & Estimación & $\%$ de la población & Fuente o referencia \\
\hline Personas con discapacidad & 3.528 .221 & $8,8 \%$ & - INE - EDDES, 1999. \\
\hline Personas mayores de 64 años & 6.434 .609 & $16 \%$ & - INE - EDDES, 1999. \\
\hline No discapacitadas & 4.361 .957 & $10,9 \%$ & - INE - EDDES, 1999. \\
\hline Personas sin discapacidad $^{1}$ & 7.828 .635 & $19,5 \%$ & - INE - EPA, 1999. \\
\hline Mujeres embarazadas ${ }^{2}$ & 209.475 & $0,5 \%$ & - INE - EMH, 1997. \\
\hline Discapacitados transitorios & 515.140 & $1,3 \%$ & - INE- EMH, 1997. \\
\hline Total afectados por barreras & 15.718 .813 & $39,1 \%$ & \\
\hline Total población española & 40.202 .160 & $100 \%$ & - INE - EDDES, 1999. \\
\hline
\end{tabular}

Fuente: Instituto Nacional de Estadística, documentos Encuesta sobre Discapacidades, Deficiencias y Estado de Salud (EDDES),Encuesta de Población Activa (EPA), Encuesta de Morbilidad Hospitalaria (EMH) y Libro Blanco de la Accesibilidad (Ministerio de Trabajo y Asuntos Sociales, 2002 y 2003).

A la hora de determinar los turistas potenciales beneficiarios directamente en temas de accesibilidad, se encuentran diferentes fuentes con distintas estimaciones. El mercado potencial para la accesibilidad ha sido estimado actualmente para 127,5 millones de beneficiarios en Europa, incluyendo en esta cifra a 7 segmentos (Buhalis et al., 2005): (i) personas con impedimentos de movilidad, (ii) personas con impedimentos visuales, (iii) personas con impedimentos auditivos, (iv) personas con impedimentos del habla, (v) personas con impedimentos mentales o intelectuales, (vi) personas con impedimentos ocultos, y (vii) personas mayores. Partiendo de los datos de las personas con discapacidad y las personas mayores, es decir, los beneficiarios directos de accesibilidad, representarían casi la quinta parte de la población mundial, entre 1,3 y 1,6 billones. Las personas mayores y las personas con discapacidad, ambas beneficiarias directas de la accesibilidad y colectivos vinculados explícitamente (Burnett, 1996; Burnett y Baker, 2001; Fuguet, 2008), representan más de la quinta parte de la población mundial. Además, cada vez 
son más los consumidores con discapacidad y personas mayores que demandan bienes y servicios turísticos. Según datos de la Comisión Europea (1997), se barajarían cifras que rondarían los 8 millones de turistas europeos con discapacidad que viajarán al extranjero, 15 millones que viajarán dentro del país, 22 millones de excursiones locales de un día y todos ellos con una media de 0,5 acompañantes como mínimo. Esto supondría un mercado potencial de 35 millones de viajeros que pernoctarían y 630 millones de noches de hotel por año. Pero como limita el propio documento, no todas las personas están en disposición de hacer turismo, en algunos casos sus discapacidades les impiden viajar, y en otros sus condiciones económicas. En contraposición, existirían otros colectivos que serían beneficiarios de accesibilidad, como las mujeres embarazadas, personas con discapacidades transitorias físicas, personas con movilidad o comunicación reducida de forma temporal o las familias con hijos.

Teniendo en cuenta la limitación expuesta anteriormente en relación a que todas las personas con discapacidad no están en condiciones de viajar, Van Horn (2002) expone que el 70\% de europeos con diversas necesidades de accesibilidad tienen capacidad física y económica para viajar, y lo harán con una media de 0,5 acompañantes por viajero potencial. Un dato que refuerza dicha afirmación, es que de 4.000 encuestados en el estudio realizado en Alemania, el 52\% de los entrevistados viajaban con compañero (Ministerio Federal Alemán de Economía y Tecnología, 2004). Por término medio, el 59\% de las familias europeas tienen un miembro con discapacidad, y un 38\% de dicha población poseen un amigo con discapacidad (Eurobarometer, 2001). Las personas con discapacidad cogerían como media más de un período vacacional por año, viajando con más miembros de la familia o amigos, si pudieran encontrar más y mejor información sobre sitios accesibles (Buhalis et al., 2005). Si se aúnan todos los datos enumerados anteriormente, se podría hablar de un mercado beneficiario de más de 260 millones de personas con discapacidad y personas mayores principalmente, que generaría unos ingresos procedentes del turismo de 166 billones de euros (Eurostat, 2005). Dicha estimación, podría ser más alta, principalmente debido a dos causas. Primero, los cálculos están basados en la hipótesis de que los ciudadanos europeos llevarán a cabo sus vacaciones en Europa, pero hay turistas de todo el mundo que escogen Europa como destino vacacional, los cuales no han sido tenidos en cuenta anteriormente.. Segundamente, los números citados sólo se refieren a personas con discapacidad o beneficiarios directos de la accesibilidad, y como ya se ha comentado, la accesibilidad beneficia a todos los usuarios (Buhalis et al., 2005).

Con los datos recogidos, parece incomprensible la ignorancia del mercado hacia el colectivo de discapacitados. Nadine Vogel (2006) esgrime principalmente tres razones que pueden explicar este hecho: las personas están, por lo general, incómodas con los discapacitados, las necesidades especiales de estos colectivos son consideradas como un "nicho" y las empresas tienen miedo a "hacerlo mal". Así, la falta de accesibilidad de la industria turística en relación a sus bienes, servicios y entornos, pueden derivar en tres supuestos, (Franco, 1999):

- Por lo general, la accesibilidad es un elemento de calidad, por lo que si no se dispone de ella, el servicio ofrecido es de menor calidad.

- Si el sector (industria, establecimiento, bien, servicio o destino) no se plantea dicho segmento de mercado como propio y no se instauran y cumplen normas de accesibilidad, se están desperdiciando oportunidades de negocio entre potenciales turistas (y sus acompañantes) con diversas disfuncionalidades.

- Si tomando el caso contrario, afirman ser accesibles e inciden en esta ventaja competitiva, pero realmente no lo son, asumen costes de no calidad.

\section{Importancia del turismo accesible en España}

Para identificar a los beneficiarios de accesibilidad, así como determinar los potenciales ingresos que podrían generar para el sector en España, se toma como referencia los trabajos realizados por Domínguez et al. (2013) y Alén et al. (2012) que utilizaron el modelo propuesto por Eurostat (2005). Como se observa en la tabla 3, se generan 4 escenarios diferenciados en base al efecto multiplicador del acompañante, así como de la duración media del viaje, que para el segmento de turistas españoles con discapacidad se establece en 5 o 10 días. Por tanto, los ingresos potenciales oscilarían entre los casi 8.000 millones de euros en el caso más negativo, a los 31.881 millones en el más positivo. 
Tabla 3: Mercado potencial de turistas españoles beneficiarios de la accesibilidad e ingresos

\begin{tabular}{|c|c|c|c|c|c|c|c|}
\hline $\begin{array}{c}\text { Demanda } \\
\text { general de } \\
\text { accesibilidad }\end{array}$ & $\begin{array}{c}70 \% \text { posee } \\
\text { capacidad } \\
\text { física y } \\
\text { económica }\end{array}$ & $\begin{array}{c}\text { Efecto } \\
\text { múltiple de } \\
\text { amigos y }\end{array}$ & $\begin{array}{l}\text { Compañía } \\
\text { de amigos y } \\
\text { familiares }\end{array}$ & $\begin{array}{c}\text { TOTAL } \\
\text { mercado } \\
\text { potencial de }\end{array}$ & \multicolumn{2}{|c|}{$\begin{array}{c}\text { Media de gasto por } \\
\text { vacaciones }\end{array}$} & $\begin{array}{c}\text { Ingresos } \\
\text { potenciales } \\
\text { del turismo }\end{array}$ \\
\hline \multirow{4}{*}{$\begin{array}{c}15,7 \\
\text { millones }\end{array}$} & \multirow{4}{*}{$\begin{array}{c}10,99 \\
\text { millones }\end{array}$} & \multirow{2}{*}{0,5} & \multirow{2}{*}{$\begin{array}{c}5,49 \\
\text { millones }\end{array}$} & \multirow{2}{*}{$\begin{array}{c}16,48 \\
\text { millones }\end{array}$} & \multirow{4}{*}{$96,70 €$} & 10 días & $\begin{array}{l}15.936,16 \\
\text { millones } €\end{array}$ \\
\hline & & & & & & 5 días & $\begin{array}{l}7.968,08 \\
\text { millones } €\end{array}$ \\
\hline & & \multirow{2}{*}{2} & \multirow{2}{*}{$\begin{array}{c}21,98 \\
\text { millones }\end{array}$} & \multirow{2}{*}{$\begin{array}{c}32,97 \\
\text { millones }\end{array}$} & & 10 días & $\begin{array}{l}31.881,99 \\
\text { millones } €\end{array}$ \\
\hline & & & & & & 5 días & $\begin{array}{l}\text { 15.936,16 } \\
\text { millones } €\end{array}$ \\
\hline
\end{tabular}

Fuente: Domínguez et al, 2013.

Tanto en el escenario más negativo, como en el más positivo, la rentabilidad estimada es lo suficientemente representativa como para realizar un esfuerzo por parte de las organizaciones públicas y privadas para aprovechar la oportunidad que se genera, ampliando el mercado y trabajando en base a la calidad y la responsabilidad social.

\section{El turista español con discapacidad}

A la hora de analizar al turista con discapacidad, es fundamental determinar tanto su perfil como hábitos de comportamiento, dado que facilitará poder establecer las claves para los agentes turísticos, y concretamente para las organizaciones que gestionan alojamientos, que es nuestro foco de estudio.

De nuevo se toman como base los trabajos de Domínguez et al. (2011) y Alén et al. (2012), realizando un análisis generalizado al descartar aquellas respuestas no mayoritarias, siendo posible establecer cuatro grupos de perfiles de turistas españoles con discapacidad (ver Tabla 4).

Destaca que las personas menores de 65 años poseen una mayor nivel educativo, trabajan y poseen una renta familiar de entre 1.200 y $3.000 €$. A todos ellos le gusta viajar muchísimo y lo hacen más de una vez al año con una duración mayor para los hombre que para las mujeres, que en algunos casos viajan de 2 a 4 días y no de 5 a 10 días. El otro gran bloque, las personas mayores de 65 años, tienen un menor nivel educativo, casi todas ellas perciben prestaciones sociales y destaca que las mujeres tienen mayor renta familiar que los hombres, hasta $3.000 €$ frente a $1.200 €$. Destacar que a las mujeres le gusta muchísimo viajar frente a los hombre a los que no les entusiasma tanto, lo hacen una vez al año con una duración de 5 a 10 días. Hacer hincapié, que la gran mayoría de encuestados poseían una discapacidad severa, es decir, entre el 50 y el $95 \%$.,

A la hora de analizar su comportamiento, se ha tenido en cuenta el tipo de discapacidad, dado que como ya se ha comentado, este factor determinará en gran medida sus hábitos. Por lo general, todos ellos suelen viajar en verano, acompañados por familiares, en busca de relax y/o actividades culturales o en la naturaleza, utilizando el coche, alojándose en hoteles y teniendo como destino mayoritario España y Europa. Las principales barreras a las que se enfrentan suelen ser arquitectónicas, urbanística y de transporte, por lo que los destinos exóticos son bastante inaccesibles y a todos ellos les gustaría que mejoraran, así como la atención del personal a las necesidades específicas derivadas de su discapacidad. Llama la atención que el colectivo de discapacidad física tiende a comportamientos similares a los de discapacidad oculta.

\section{Discapacidad y hospedaje}

Las personas con discapacidad deben localizar un alojamiento adecuado que facilite y posibilite el viajar, ya que estar alejado de su residencia habitual requiere que tanto la habitación como el baño sean accesibles según su discapacidad (Dwyer y Darcy, 2008). Pero aunque dicho elemento es fundamental, 
Tabla 4: Segmentación, comportamiento y hábitos de los turistas españoles con discapacidad

\begin{tabular}{|c|c|c|c|c|}
\hline SEGMENTO & GRUPO 1 & GRUPO 2 & GRUPO 3 & GRUPO 4 \\
\hline Sexo & Mujer & Hombre & Mujer & Hombre \\
\hline Edad & \multicolumn{2}{|c|}{ Menores de 65 años } & \multicolumn{2}{|c|}{ Mayores de 65 años } \\
\hline Estado civil & Casada & Soltero & \multicolumn{2}{|c|}{ Casado } \\
\hline Nivel de estudios & $\begin{array}{c}\text { Estudios } \\
\text { primarios o } \\
\text { universitarios }\end{array}$ & $\begin{array}{c}\text { Estudios FP } \\
\text { I, FP II o } \\
\text { universitario }\end{array}$ & \multicolumn{2}{|c|}{ Estudios primarios } \\
\hline Situación laboral & $\begin{array}{c}\text { Trabajador } \\
\text { en activo o } \\
\text { prestación social }\end{array}$ & $\begin{array}{l}\text { Trabajador en } \\
\text { activo }\end{array}$ & \multicolumn{2}{|c|}{ Percibe prestación social } \\
\hline $\begin{array}{l}\text { Ingresos mensuales } \\
\text { núcleo familiar }\end{array}$ & \multicolumn{2}{|c|}{ Entre $1.200 €$ y $3.000 €$} & Hasta $3.000 €$ & Hasta $1.200 €$ \\
\hline Tipo de discapacidad & Física u oculta & Oculta o física & Física u oculta & Oculta o física \\
\hline Grado de discapacidad & \multicolumn{2}{|c|}{ Severo (del 50\% al 95\%) } & \multicolumn{2}{|c|}{ Severo (del 50\% al 95\%) } \\
\hline Uso de ayudas & \multicolumn{2}{|c|}{ Ninguna o silla de ruedas } & \multicolumn{2}{|c|}{$\begin{array}{c}\text { Ninguna o bastones, muletas y } \\
\text { similares }\end{array}$} \\
\hline Gusto por viajar & \multicolumn{2}{|c|}{ Mucho } & Muchísimo & $\begin{array}{l}\text { Entre regular y } \\
\text { mucho }\end{array}$ \\
\hline Frecuencia de viaje & \multicolumn{2}{|c|}{ Más de una vez al año } & \multicolumn{2}{|c|}{ Una vez al año } \\
\hline Duración viaje & $\begin{array}{l}\text { De } 2 \text { a } 4 \text { días o } \\
\text { de } 5 \text { a } 10 .\end{array}$ & De 5 a 10 días & De 5 & días \\
\hline
\end{tabular}

Fuente: Domínguez et al., 2011. 


\begin{tabular}{|c|c|c|c|c|}
\hline COMPORTAMENTO & FÍSICA & PSIQUICA & SENSORIAL & OCULTA \\
\hline Preferencia para viajar & \multicolumn{4}{|c|}{ Verano } \\
\hline $\begin{array}{c}\text { Razón para no viajar en } \\
\text { esas fechas }\end{array}$ & $\begin{array}{l}\text { Económicas y } \\
\text { masificación } \\
\text { turística }\end{array}$ & $\begin{array}{c}\text { Falta de } \\
\text { acompañante }\end{array}$ & $\begin{array}{l}\text { Razones } \\
\text { económicas }\end{array}$ & $\begin{array}{c}\text { Económicas y de } \\
\text { trabajo }\end{array}$ \\
\hline Formas como suele viajar & \multicolumn{4}{|c|}{ Familiares } \\
\hline $\begin{array}{c}\text { Por qué escoge esa forma } \\
\text { de viaje }\end{array}$ & \multicolumn{4}{|c|}{ Por comodidad y por ir con gente conocida } \\
\hline $\begin{array}{c}\text { Toma de decisiones sobre } \\
\text { el viaje a realizar }\end{array}$ & $\begin{array}{l}\text { Familia y la } \\
\text { propia persona }\end{array}$ & Familia & $\begin{array}{l}\text { Propia persona y } \\
\text { la familia }\end{array}$ & Persona \\
\hline $\begin{array}{c}\text { Destino que le gustaría } \\
\text { visitar }\end{array}$ & España & $\begin{array}{l}\text { España y resto } \\
\text { del mundo }\end{array}$ & Resto del mundo & $\begin{array}{c}\text { Resto del } \\
\text { mundo, España } \\
\text { y Europa }\end{array}$ \\
\hline $\begin{array}{c}\text { Atractivos que suelen } \\
\text { escoger }\end{array}$ & Playa & $\begin{array}{l}\text { Pequeñas } \\
\text { poblaciones }\end{array}$ & $\begin{array}{l}\text { Patrimonio } \\
\text { y grandes } \\
\text { ciudades }\end{array}$ & Playa \\
\hline $\begin{array}{l}\text { Les gustaría que se } \\
\text { realizar un mayor } \\
\text { esfuerzo }\end{array}$ & \multicolumn{4}{|c|}{ Destinos exóticos, de patrimonio y en las grandes ciudades } \\
\hline $\begin{array}{l}\text { Actividades que les gusta } \\
\text { realizar }\end{array}$ & $\begin{array}{l}\text { Actividades } \\
\text { culturales y de } \\
\text { relax }\end{array}$ & $\begin{array}{l}\text { Actividades en } \\
\text { la naturaleza y } \\
\text { relax }\end{array}$ & Relax & $\begin{array}{l}\text { Actividades } \\
\text { culturales y } \\
\text { relax }\end{array}$ \\
\hline Gasto medio & \multicolumn{4}{|c|}{ Entre 500 y 750 en viajes de 5 días } \\
\hline Transporte & Coche familiar & $\begin{array}{l}\text { Coche familiar y } \\
\text { autobús }\end{array}$ & $\begin{array}{l}\text { Coche familiar, } \\
\text { tren y autobús }\end{array}$ & $\begin{array}{l}\text { Coche propio y } \\
\text { familiar }\end{array}$ \\
\hline Hospedaje & \multicolumn{4}{|c|}{ Hotel } \\
\hline Fuentes de información & Familia & $\begin{array}{c}\text { Familia y } \\
\text { asociaciones }\end{array}$ & Familia y amigos & Familia y amigos \\
\hline Valoración del personal & $\begin{array}{l}\text { Entre moderada } \\
\text { y bastante }\end{array}$ & Bastante & $\begin{array}{l}\text { Entre poca y } \\
\text { moderada }\end{array}$ & $\begin{array}{l}\text { Entre moderada } \\
\text { y bastante }\end{array}$ \\
\hline $\begin{array}{c}\text { Mejorar en formación del } \\
\text { personal }\end{array}$ & \multicolumn{4}{|c|}{ Atención necesidades específicas } \\
\hline Principales barreras & $\begin{array}{l}\text { Arquitectónica, } \\
\text { urbanísticas y de } \\
\text { transporte }\end{array}$ & $\begin{array}{l}\text { Humanas, } \\
\text { arquitectónica y } \\
\text { urbanísticas }\end{array}$ & $\begin{array}{l}\text { Comunicación y } \\
\text { transporte }\end{array}$ & $\begin{array}{l}\text { Arquitectónica, } \\
\text { humanas, } \\
\text { urbanas y de } \\
\text { transporte }\end{array}$ \\
\hline
\end{tabular}


no existen muchas investigaciones, destacando la realizada por Darcy (2010) sobre los criterios de accesibilidad utilizados, la información disponible y la coherencia entre ambas según los turistas con discapacidad que utilizan hoteles en Australia; Israeli (2002) que realizó un trabajo previo sobre los elementos base que deben darse conjuntamente para que un sitio sea accesible; o a nivel nacional, los desarrollados por Fernández (2007) que se centran en el análisis de accesibilidad hotelera de la provincia de Cádiz.

La información existente sobre turismo accesible y alojamiento, por lo general, se centra principalmente en la normativa existe que regula dicha actividad. En España destaca el Real Decreto 556/1989 del 19 de mayo sobre atribución de medidas mínimas sobre accesibilidad en los edificios, complementado posteriormente con la Ley 15/95 del 30 de mayo sobre límites del dominio sobre inmuebles para eliminar barreras arquitectónicas a las personas con discapacidad y el reglamento sobre los requisitos mínimos de infraestructuras en los alojamientos turísticos. Es importante recordar que las competencias sobre discapacidad y accesibilidad están transferidas a las distintas Comunidades Autónomas, lo cual hace que tengan diferentes niveles de desarrollo en relación a la legislación sobre discapacidad, y concretamente sobre turismo accesible. Por lo general, en las Comunidades Autónomas no existen normativas específicas sobre este último elemento, el turismo accesible, aunque si se hace mención a la no discriminación entre personas a causa de su discapacidad, y el cumplimiento de la normativa existente sobre accesibilidad en los edificios, aunque no se especifican los criterios técnicos. La tendencia es que sólo se recoga el número de habitaciones "adaptadas" o "polivalentes" que deben disponer según el número total de habitaciones que tengan, que obviamente dista mucho según la Comunidad Autónoma a la que se haga referencia, por ejemplo, para tener 2 habitaciones adaptadas o polivalentes: en Andalucía los establecimientos deben ser de entre 75 y 150 habitaciones, en Murcia de entre 101 y 250, y en Cataluña de entre 101 y 150. Queda clara la disparidad existente entre normativas.

Para otro tipo de alojamientos, existen normativas en algunas comunidades a nivel de regulación en materia de accesibilidad, como es el caso de los albergues, pero muy diferenciada y focalizada en temas arquitectónicos y de señalización.

Todo lo expuesto hasta ahora queda reflejado en los escasos datos existentes sobre accesibilidad de los alojamientos en España, donde destaca que sólo el 16\% de las casas rurales son accesibles para personas con movilidad reducida localizadas principalmente en País Vasco y Galicia, con un 37\% y un33\% de la oferta disponible respectivamente (Hosteltur, 2013). En lo tocante a los campings, y según un estudio realizado por Eroski Consumer (2011) que analizó 100 campings de 18 Comunidades Autónomas de un total de 1.168 ( 24 de $1^{a}$ categoría, 64 de $2^{\text {a }}$ categoría y los restantes de $3^{\text {a }}$ categoría), los resultados mostraron que 40 de ellos no estaban adaptados, 31 de ellos sólo se centraron en la accesibilidad de los servicios, y de esos, 4 habían tenido en cuenta la accesibilidad al restaurante, piscina y recepción. Sólo 25 de ellos estaban totalmente adaptados para acoger a personas con discapacidad física, y ninguno para las personas con discapacidad visual. Sobre el alojamiento en hoteles, no existen cifras en concreto aunque si están disponibles listas de los hoteles con mayor accesibilidad, donde destaca la cadena hotelera Confortel, la cual ya posee en todos sus hoteles la Certificación de Sistemas de Gestión de Accesibilidad Universal de AENOR. Para finalizar con este punto, comentar que la accesibilidad en balnearios, aunque no está cuantificada, si tiende a ser mayor dado los programas sociales existentes que promueven las Administraciones Pública a través de ofertas de plazas para dicho colectivo

\section{Objetivo y metodología del estudio}

Uno de los datos clave para el planteamiento de dicho estudio, parte de la estimación de la Comisión Europea (1997), que cifraba que los turistas europeos con discapacidad disfrutarían de 630 millones de noches de hotel por año. A dicha cifra, bastante representativa por sí sola, habría que añadirle todas aquellas pernoctas que realizarían en otros alojamientos. Por todo ello, el objetivo de este estudio, es determinar la tipología de hospedaje utilizado por los turistas españoles con discapacidad, así como las variables influyen en su comportamiento a nivel sociodemográfico (sexo, edad, tipo de discapacidad) y comportamental (duración de la estancia, frecuencia anual de viajes, destino seleccionado).

Para dar cumplimiento a dicho objetivo, se parte de la premisa que las personas con discapacidad son uno de los usuarios con mayor rango de necesidades y por lo tanto, de exigencias, por lo que los resultados se presentan según tipología de discapacidad. El universo a analizar fueron los turistas españoles con algún tipo de discapacidad, siendo el ámbito geográfico España, con un tamaño muestral de 404 encuestas válidas y un nivel de confianza del 95,5\%. Tomándose como base los datos del INE 
(1999) y Eurostat (2005), se establecieron cuatro elementos clave para estructurar la muestra: sexo (hombre, mujer), edad (17 a 64 años, 65 y más años), Comunidad Autónoma (20 en total) y tipología de discapacidad (física, psíquica, sensorial y oculta), por lo que se utilizó un muestreo por cuotas que divide a la población en distintas subpoblaciones, al tener que combinar datos de fuentes diferentes. Dichos datos fueron recogidos a través de entrevista personal del 1 de agosto al 30 de septiembre de 2008.

\section{Análisis de resultados}

Según los datos analizados, inicialmente se debe hacer mención a que los turistas españoles con discapacidad física o oculta consideran que las peores barreras existentes son las arquitectónicas seguidas de las urbanas y de las de transporte; para el colectivo con discapacidad psíquica, son tan importantes las arquitectónicas como las humanas; y para las personas con discapacidad sensorial, primero son las comunicativas seguidas de las de transporte, arquitectónicas y humanas (ver Gráfico 1). Tomando como referencia dichos datos, se constata que las barreras arquitectónicas son uno de los mayores impedimentos para los turistas con discapacidad aunque no es el principal obstáculos a la hora de viajar (ver Gráfico 1), donde destacan los problemas de comunicación y aquellos vinculados a la realización de actividades, excursiones o desplazamientos, estando el hospedaje considerado como uno de los obstáculos con menor influencia para todos los colectivos. Dicho dato es caro, dado que junto al transporte para llegar al destino, son los elementos clave para el consumo de productos turísticos accesibles, y por lo tanto, son a los que se le dedica más tiempo en la búsqueda de información específica en relación a su accesibilidad.

\section{Gráfico 1: Barreras y obstáculos para los turistas con discapacidad}

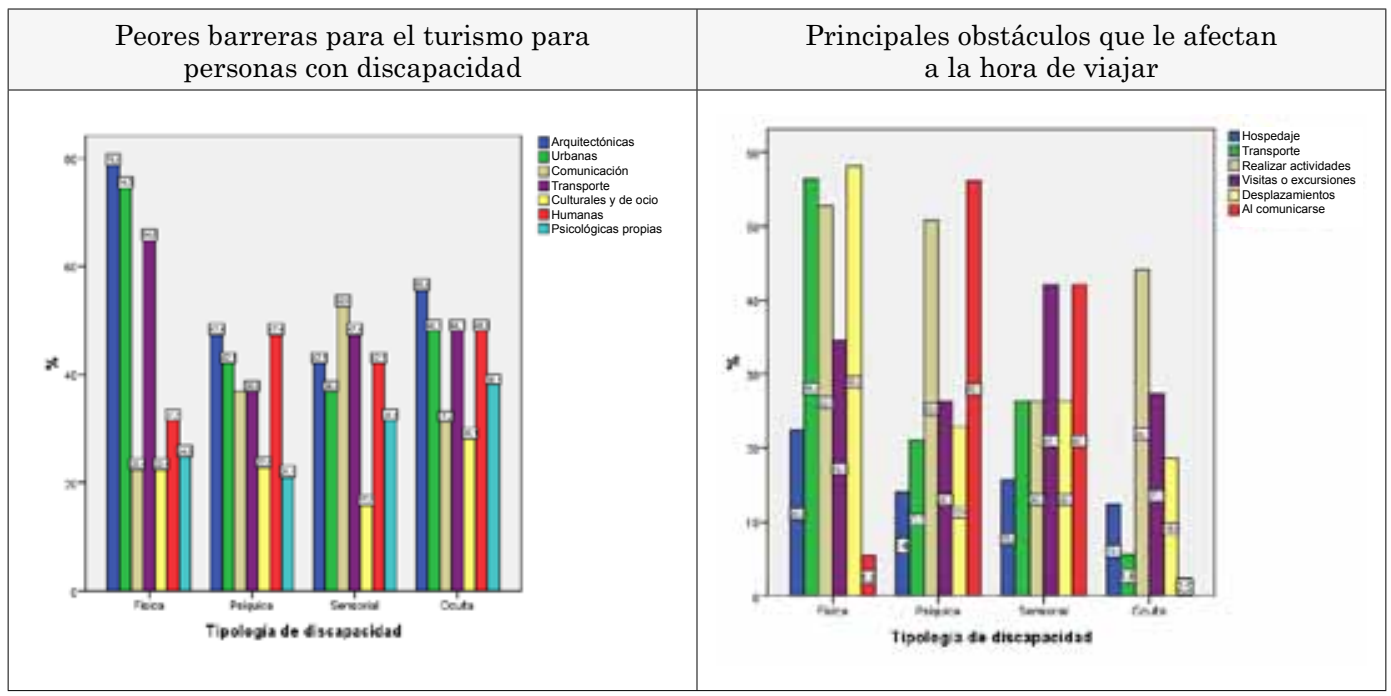

Tomando como foco el análisis específico sobre las preferencias de alojamiento (ver Gráfico 2), habitualmente todos los colectivos optan por hoteles y secundariamente por residencias propias o segundas casas, a excepción del colectivo con discapacidad sensorial que utiliza apartamentos en vez de segunda residencia. Los hostales y pensiones, así como los camping, tienen un uso bajo a excepción de las personas con discapacidad oculta, preferentemente a causa de su grado de accesibilidad. Reseñar que los albergues son un hospedaje bastante utilizado por todos los colectivos.

Analizando y valorando el grado de accesibilidad de dichos alojamientos, reseñar que los hostales y pensiones están consideradas como bastante accesibles o muy accesibles por el 30,7\% de la muestra analizada; la residencia propia también es valorada como bastante o muy accesible por un $45,3 \%$ ya que hay que tener en cuenta que por lo general estas segundas residencias suelen ser pisos que están condicionados por el diseño arquitectónico de los portales y los diferentes niveles. Los balneario rondan 
el 40\%, mientras que los camping están considerados como bastante o muy accesible sólo por el 16,8\% de la muestra analizada, situación similar a la de los albergues que obtienen un porcentaje cercano al $21,7 \%$ en valoración de hospedaje bastante o muy accesible. Todos estos resultados están sujetos a que una gran parte de la muestra analizada posee discapacidad oculta, la cual no supone restricciones tan importantes como las físicas, psíquicas o sensoriales, teniendo que enfrentarse a un número bastante más reducido de barreras.

\section{Gráfico 2: Barreras y obstáculos para los turistas con discapacidad}

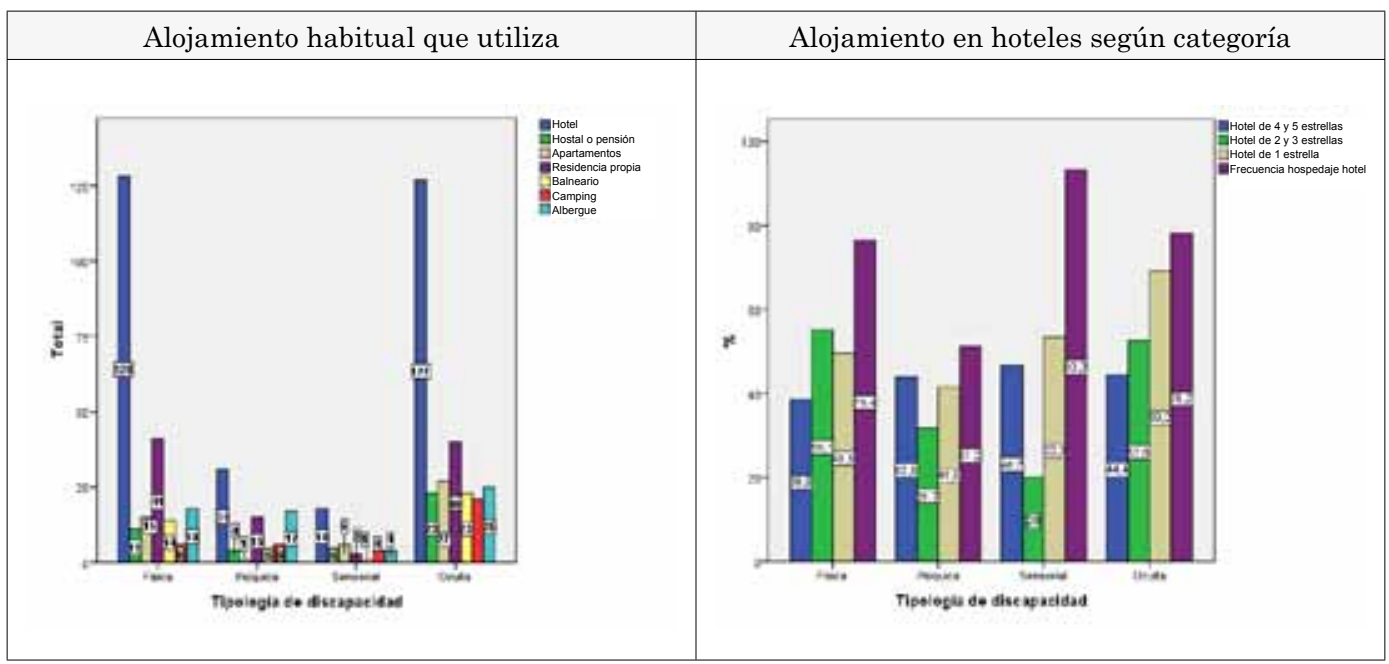

Dado que el hotel, es el tipo de alojamiento más utilizado, se realiza un análisis más exhaustivo (ver Gráfico 2). Las personas con discapacidad física utilizan preferentemente hoteles de 2 y 3 estrellas seguidos por los de 1 , un hecho peculiar ya que los hoteles más adaptados y accesibles son los de $4 \mathrm{y}$ 5 estrellas, -un $65,8 \%$ de los encuestados los valora como bastante accesibles o muy accesibles-, y el colectivo de discapacidad física es uno de los que más barreras arquitectónicas padece. En lo tocante a las personas con discapacidad psíquica, preferentemente recurren a hoteles de 4 y 5 estrellas, seguidos por los de 1 estrella. Destacar que éstos últimos fueron valorados como bastante o muy accesibles por un $35,8 \%$ de la muestra analizada. Se pasa de un extremo a otro, al igual que con el colectivo de personas con discapacidad sensorial, en la que se opta primeramente por hoteles de 1 estrella y de forma muy cercana en porcentaje, a hoteles de 4 y 5 estrellas.

Una vez establecido sus hábitos en el tipo de alojamiento, se estudia que variables afectan a dicho comportamiento, por lo que se plantea como objetivo: determinar si la variación del número de viajes realizados el último año se ve condicionada por el tipo de hospedaje utilizado, la edad, la tipología de discapacidad y el gasto medio por día del viaje.

El nivel crítico asociado al estadístico $F$ del modelo corregido (ver Tabla 5), indica que el modelo explica una parte significativa de la variación observada en la variable dependiente número de viajes realizados el último año. Existe un efecto interacción que surge derivado de la combinación de las diferentes variables, en concreto, la relación se da entre tipo de discapacidad y hospedaje utilizado, poseyendo un efecto significativo sobre el número de viajes realizados el último año. Dichas variables, cuando se analizan los efectos principales, se observa que ambas difieren, con valores inferiores al p-valor. Esto es, que los grupos definidos por las variables hospedaje y tipología de discapacidad, tanto de forma individualizada o mediante combinación mutua, distan del número medio de viajes realizados el último año. Situación opuesta a las combinaciones con las variables sexo y edad, las cuales no son significativas. 
Tabla 5: Pruebas de los efectos inter-sujetos

\begin{tabular}{|c|c|c|c|c|c|}
\hline Fuente & $\begin{array}{l}\text { Suma de } \\
\text { cuadrados }\end{array}$ & gl & $\begin{array}{c}\text { Media } \\
\text { cuadrática }\end{array}$ & $\mathbf{F}$ & Significación \\
\hline Modelo corregido & $133,048^{\mathrm{a}}$ & 76 & 1,751 & 1,881 & , 000 \\
\hline Intersección & 359,003 & 1 & 359,003 & 385,703 & 000 \\
\hline Tipología de discapacidad & 12,048 & 3 & 4,016 & 4,315 & ,005 \\
\hline Hospedaje & 22,210 & 6 & 3,702 & 3,977 & ,001 \\
\hline Sexo & ,872 & 1 & ,872 & ,937 & 334 \\
\hline Edad recodificada & 1,663 & 1 & 1,663 & 1,787 & , 182 \\
\hline Tipo.disc * Hospedaje & 28,231 & 14 & 2,016 & 2,166 & ,009 \\
\hline Tipo.disc * sexo & 2,669 & 3 & 890 & ,956 & ,414 \\
\hline Tipo.disc * rec_edad & 1,186 & 3 & ,395 & 425 & ,735 \\
\hline Hospedaje ${ }^{*}$ sexo & 5,579 & 6 & ,930 & ,999 & ,426 \\
\hline Hospedaje * rec_edad & 4,576 & 6 & ,763 & ,819 &, 556 \\
\hline Sexo * rec_edad &, 320 & 1 &, 320 & ,343 &, 558 \\
\hline Tipo.disc * Hospedaje * sexo & 12,526 & 11 & 1,139 & 1,223 & ,270 \\
\hline Tipo.disc * Hospedaje * rec_edad & 2,476 & 9 & ,275 & ,296 & ,976 \\
\hline Tipo.disc ${ }^{*}$ sexo ${ }^{*}$ rec_edad & 2,961 & 3 & ,987 & 1,060 & ,366 \\
\hline Hospedaje ${ }^{*}$ sexo ${ }^{*}$ rec_edad & 2,753 & 4 & ,688 & ,739 &, 566 \\
\hline $\begin{array}{l}\text { Tipo.disc * Hospedaje * sexo * } \\
\text { rec_edad }\end{array}$ & 6,982 & 5 & 1,396 & 1,500 &, 189 \\
\hline Error & 283,886 & 305 & ,931 & & \\
\hline Total & 1925,000 & 382 & & & \\
\hline Total corregida & 416,935 & 381 & & & \\
\hline
\end{tabular}

Variable dependiente: número de viajes realizados el último año.

Mediante la aplicación del contraste de Levene mostrado en la tabla 6, se rechaza la hipótesis nula de igualdad de que la varianza error de la variable dependiente número de viajes realizados el último año, es igual a lo largo de todos los grupos.

Tabla 6: Contraste de Levene sobre la igualdad de las varianzas error

\begin{tabular}{|c|c|c|c|}
\hline $\mathbf{F}$ & gl1 & gl2 & Significación \\
\hline 1,618 & 76 & 305 & ,002 \\
\hline
\end{tabular}

Partiendo de los efectos principales que resultaron significativos, así como de la interacción que se da entre ambos, se aplica una comparación post hoc para determinar dónde se encuentra las diferencias de dichos promedios. Se aprecia que las personas con discapacidad sensorial en relación al número medio de viajes realizados el último año, difiere significativamente de las personas con discapacidad oculta y psíquica. Además este último colectivo tiene un comportamiento muy similar a al colectivo de discapacidad oculta. Dicho hecho se refleja en la tabla 7 en la que se aprecian dos subconjuntos claros, por un lado se agrupan los colectivos de discapacidad física, psíquica y oculta, dejando en el otro subconjunto a las personas con discapacidad sensorial, las cuales tienen un número medio de viajes realizados el último año superior al resto. 
Tabla 7: Subconjuntos homogéneos

\begin{tabular}{|l|l|r|r|r|}
\hline & \multicolumn{1}{|c|}{$\begin{array}{c}\text { Tipología de } \\
\text { discapacidad }\end{array}$} & \multirow{2}{*}{ N } & \multicolumn{2}{|c|}{ Subconjunto } \\
\cline { 3 - 5 } & Psíquica & 52 & $\mathbf{1}$ & \multicolumn{1}{|c|}{$\mathbf{2}$} \\
\cline { 2 - 5 } & Oculta & 156 & 1,83 & \\
\cline { 2 - 5 } DHS de Tukey & Física & 155 & 2,10 & 2,68 \\
\cline { 2 - 5 } & Sensorial & 19 & & 1,000 \\
\cline { 2 - 5 } & Significación & &, 526 & \\
& & & & \\
\end{tabular}

En la siguiente tabla, se observa que la utilización del hotel en relación al número medio de viajes realizados el último año dista significativamente de la utilización de albergue, y éste a su vez del uso del balneario. Es decir, en relación al número de viajes realizados el último año si se escoge un hotel o balneario para alojarse, no se utilizan albergues, y viceversa. Situación opuesta se da en la utilización casi indistinta si se escoge como alojamiento hotel, hostal o pensión, apartamento, residencia propia o balneario en relación al número de medio de viajes realizados el último año; aunque no se establecen subconjuntos homogéneos al agruparse todos en el mismo, como se aprecia en la tabla 9 .

Tabla 8: Comparaciones múltiples

\begin{tabular}{|c|c|c|c|c|c|}
\hline & (I) Hospedaje & $\begin{array}{c}(\mathrm{J}) \\
\text { Hospedaje }\end{array}$ & $\begin{array}{c}\text { Diferencia } \\
\text { entre } \\
\text { medias (I-J) }\end{array}$ & Error típ. & Significación \\
\hline \multirow{18}{*}{$\begin{array}{l}\text { Games- } \\
\text {-Howell }\end{array}$} & \multirow{6}{*}{ Hotel } & Hostal o pensión & ,05 & 283, & 1,000 \\
\hline & & Apartamento &,- 02 & ,223 & 1,000 \\
\hline & & Residencia propia &,- 05 &, 141 & 1,000 \\
\hline & & Balneario & , 14 & ,156 & ,974 \\
\hline & & Camping &,- 66 & ,399 & ,658 \\
\hline & & Albergue &,$- 50^{*}$ & ,167 &, 050 \\
\hline & \multirow{6}{*}{ Balneario } & Hotel &,- 14 & , 156 & ,974 \\
\hline & & Hostal o pensión &,- 08 & ,301 & 1,000 \\
\hline & & Apartamento &,- 16 &, 247 & ,994 \\
\hline & & Residencia propia &,- 19 & ,175 & ,935 \\
\hline & & Camping &,- 80 & ,412 & ,497 \\
\hline & & Albergue &,$- 64^{*}$ & , 197 &, 025 \\
\hline & \multirow{6}{*}{ Albergue } & Hotel &, $50^{*}$ & , 167 & ,050, \\
\hline & & Hostal o pensión &, 56 & ,307 &, 556 \\
\hline & & Apartamento & , 48 &, 254 &, 501 \\
\hline & & Residencia propia & , 45 & , 185 & , 187 \\
\hline & & Balneario &, $64^{*}$ & ,197 &, 025 \\
\hline & & Camping &,- 15 & ,416 & 1,000 \\
\hline
\end{tabular}


Tabla 9: Subconjuntos homogéneos

\begin{tabular}{|l|l|r|r|}
\hline \multicolumn{1}{|c|}{ Hospedaje } & \multirow{2}{*}{ N } & \multicolumn{2}{c|}{ Subconjunto } \\
\cline { 2 - 4 } & Balneario & 40 & $\mathbf{1}$ \\
\cline { 2 - 4 } & Hostal o pensión & 12 & 1,75 \\
\cline { 2 - 4 } DHS de Tukey $^{\text {a }}$ & Hotel & 152 & 1,83 \\
\cline { 2 - 4 } & Apartamento & 23 & 1,89 \\
\cline { 2 - 4 } & Residencia propia & 80 & 1,91 \\
\cline { 2 - 4 } & Albergue & 64 & 1,94 \\
\cline { 2 - 4 } & Camping & 11 & 2,39 \\
\cline { 2 - 5 } & Significación & & 2,55 \\
\hline
\end{tabular}

Por último, observando el gráfico de perfil de los dos factores principales y su interacción (ver Gráfico 3), los cuales poseen comportamientos diferenciados, se puede determinar que el uso del hotel como alojamiento en relación al número medio de viajes realizados el último año posee una frecuencia media muy similar para los diferentes colectivos según discapacidad, aunque es un poco superior para los grupos de personas con discapacidad física y oculta. Similar comportamiento puede ser identificado en el uso de residencia propia, que aún siendo bastante homogéneo destaca principalmente el colectivo de discapacidad psíquica seguido por los físicos, sensoriales y ocultos, pero con menor frecuencia media.

\section{Gráfico 3: Perfil tipología de discapacidad - hospedaje}

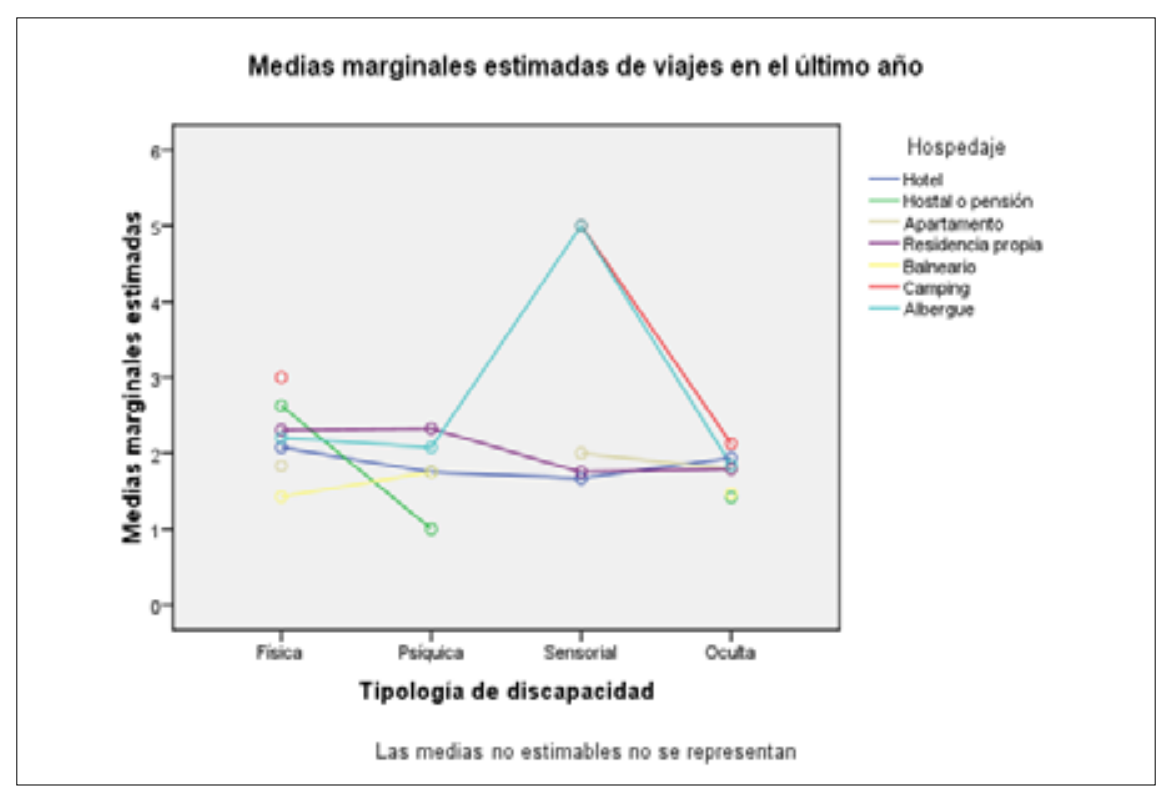

Si se centran los resultados según colectivo, destaca que:

Las personas con discapacidad física, en relación al número medio de viajes realizados el último año, utilizan el camping como hospedaje con una alta frecuencia media, que supera al resto de alojamientos, aunque está seguida de cerca por el uso de hostales y pensiones (son el colectivo que tienen una mayor frecuencia de uso-, residencia propia), albergues y apartamentos, quedando relegado a una posición menos importante la utilización de balnearios. 
El colectivo de discapacitados psíquicos no suele utilizar ni apartamentos ni camping, aunque sí utiliza albergues, pero con una frecuencia media inferior al resto de colectivos en relación al número medio de viajes realizados el último año. Destaca que es el colectivo que menos emplea el hostal o pensión, aunque en contraposición es el que más utiliza el balneario.

El grupo de discapacitados sensoriales, en relación al número medio de viajes realizados el último año, escogen como medios de hospedaje los hoteles, la residencia propia y los apartamentos, con frecuencias medias muy parejas, pero en cambio, no utilizan ni balnearios, ni hostales o pensiones. Llama la atención que con bastante diferencia son los que mayor frecuencia media tienen en el uso de camping y albergues cuanto más viajan, lo cual establece un perfil bastante claro del tipo de turismo y hospedaje preferente.

Las personas con discapacidad oculta tienen frecuencias medias muy similares y cercanas en relación al número medio de viajes realizados el último año, donde la más alta es el camping, seguida de albergues y hoteles casi al mismo nivel, residencia propia, y con valores un poco menores, los balnearios y hostales o pensiones.

\section{Conclusiones y limitaciones}

La oportunidad de negocio que se genera a través de turismo accesible, y de aquellos colectivos beneficiarios de accesibilidad, es clara, permitiendo ampliar la oferta nuevos segmento, desestacionalizar el sector dado que estos colectivos poseen mayor disponibilidad temporal, además de hacer una clara apuesta hacia productos y servicios de alta calidad basados en la accesibilidad entendida como usabilidad por y para todos. A parte de todo ello, es fundamental recordar que todo el mundo tiene derecho a disfrutar de su tiempo de ocio y tiempo libre, así como las tendencias demográficas y de la propia OMT (2011), que señalan un envejecimiento poblacional, aumento de turistas de la $3^{\mathrm{a}}$ edad, así como la búsqueda de nuevas experiencias.

Aunque en general el hotel es el alojamiento más utilizado por los diferentes colectivos según tipología de discapacidad, este se ve influenciado según la frecuencia media de viajes realizados en el último año. Dicho comportamiento viene respaldado al ser el alojamiento mejor valorado en accesibilidad según los encuestados, diferenciando dos claras tendencias, los que se alojan en hoteles de 1 a 3 estrellas, y aquellos que lo hacen en los de 4 o 5 estrellas. Pero cuando la frecuencia media de viajes anuales se incrementa, se tiende a otro tipo de alojamiento, el cual varía según la tipología de discapacidad. En el caso de los discapacitados físicos tienden a utilizar los campings y hostales o pensiones cuando viajan sobre 3 veces al año; para el colectivo de discapacidad psíquica es la residencia o los albergues cuando viajan 2 o más veces al año; para los discapacitados sensoriales son los albergues y campings con frecuencias de viaje muy altas, cercanas a 5 viajes al año; y para los discapacitados ocultos con una frecuencia de viaje de entre 1 y 2 al año, destaca el camping y los hoteles. Este comportamiento deja claro que cuanto más se viaja, independientemente de la tipología de discapacidad, se buscan alojamientos más económicos aunque los niveles de accesibilidad sean menores, dado que como se pudo comprobar anteriormente, la accesibilidad de los campings españoles es baja, solo aprueban 1 de cada 3 (Eroski Consumer, 2011), y en relación a los albergues, sólo se deben tener en cuenta las normativas de accesibilidad en la construcción para las nuevas edificaciones.

Algunos alojamientos ya han visualizado dicha oportunidad y han establecido una ventaja competitiva sobre su competencia, pero aún existen numerosas carencias, las cuales se están corrigiendo gracias a la normativa existente sobre discapacidad y accesibilidad. Aún así, muchos de estos negocios han realizado actuaciones de accesibilidad o adaptabilidad de sus espacios que han sido ejecutadas sin tener en cuenta las directrices de expertos en la temática, por lo que en vez de mejorar han empeorado. Otro elemento negativo, es que en numerosas ocasiones la información disponible sobre el alojamiento no es la adecuada, ya que hay que tener en cuenta las diferentes tipologías de discapacidad y sus necesidades, así como tener toda la información inventariada con las medidas exactas de todos sus componentes, para poder dar respuesta a todas las necesidades que tengan este colectivo.

Para finalizar, comentar que la principal limitación derivada de este estudio es que aunque la muestra es representativa y se aglutina según tipología de discapacidad, cada individuo posee necesidades específicas, por lo que no es muy complicado poder reflejar la realidad de cada uno de ellos. Así mismo, faltaría realizar un estudio exhaustivo de la oferta que posibilitara establecer las principales carencias según las necesidades del mercado, así como mostrar de forma más clara las posibles actuaciones a realizar o puntos a mejorar. Esta es una de las líneas de investigación propuestas para el futuro. 


\section{Bibliografía}

Alén, E., Domínguez, T. y Losada, N.

2012. "Chapter 7: New opportunities for the tourism market: senior tourism and accessible tourism" in Vision for Global Tourism Industry, editado por Murat Kaumohu, Intech: 139-169.

Brown, F.

1991. "Tourism for all”. Tourism Management, Vol.12, №3: 258-260.

Buhalis D., Eichhorn, V., Michopoulou E. y Miller G.

2005. Accessibility Market and Stakeholder Analysis 2005. OSSATE/ University of Surrey, Surrey.

Buhalis, D. y Darcy, S.

2011. Accessible Tourism: Concepts and Issues. Channel View Publications, UK.

Buhalis, D., y Michopouloub, E.

2011. "Information-enabled tourism destination marketing: Addressing the accessibility market".

Current Issues in Tourism, 14(2): 145-168.

Burnett, J.J.

1996. "What services marketers need to know about the mobility-disabled consumer". The Journal of

Services Marketing, Vol. $1^{\circ}, \mathrm{N}^{\circ}$ 3: 3-20.

Burnett, J. J., y Bender-Baker, H.

2001. "Assessing the travel-related behaviors of the mobility-disabled consumer". Journal of Travel Research, 40(1): 4-11.

Chang, Y.-C., \& Chen, C.-F.

2012. "Meeting the needs of disabled air passengers: Factors that facilitate help from airlines and airports". Tourism Management, 33(3): 529-536.

Comisión Europea

1997. Accesibilidad a Turistas con Discapacidad. Manual para la industria del turismo. DG XIII. Unidad Turismo.

Comunita Research and Development Information Services (CORDIS)

1995. Cost 322: Low floor busses, final report. Disponible en http://www.cordis.lu/cost-transport/src/ cost-322.htm (consultado Agosto 2013).

Daniels, M. J., Rodgers, E. B. D., y Wiggins, B. P.

2005. "Travel tales": An interpretive analysis of constraints and negotiations to pleasure travel as experienced by persons with physical disabilities. Tourism Management, 26(6): 919-930.

Darcy, S.

2010. "Inherent complexity: Disability, accessible tourism and accommodation information preferences". Tourism Management, 31(6): 816-826.

Darcy, S.

1998. Anxiety to Access: Tourism Patterns and Experiences of New South Wales People with a physical Disability. Sydney, NSW: Tourism New South Wales.

Darcy, S., y Pegg, S.

2011. "Towards strategic intent: Perceptions of disability service provision amongst hotel accommodation managers". International Journal of Hospitality Management, 30(2): 468-476.

Domínguez, T., Fraiz, J. A., y Alén, E.

2013. "Economic profitability of accessible tourism for the tourism sector in Spain". Tourism economics http://dx.doi.org/10.5367/te.2013.0246).

Domínguez, T., Fraiz, J.A. y Alén, Mª.E.

2011. "Turismo y accesibilidad. Una vision global sobre la situación de España". Cuadernos de Turismo, $\mathrm{N}^{\mathrm{o}} 28: 23-45$.

Dwyer, L., y Darcy, S.

2011. "Chapter 14 - economic contribution of tourists with disabilities: An Australian approach and methodology". In D. Buhalis \& S. Darcy (Eds.), Accessible tourism: Concepts and issues Bristol, UK: Channel View Publications, 213-239.

Dwyer, L. y Darcy, S.

2008. "Chapter 4: Economic contribution of disability to the tourism in Australia". In S. Darcy, R. Cameron. T. Taylor, E. Womg y A. Thomson (Ed) Technical report 90040 Visitor accessibility in urban centres, 15-21.Sustainable Tourism Cooperation Research Centre. 
Eichhorn, V., Miller, G., Michopoulou, E., y Buhalis, D.

2008. "Enabling access to tourism through information schemes". Annals of Tourism Research, 35(1): 189-210.

Eroski Consumer

2011. "Uno de cada 3 campings visitados suspenden por deficiencias en seguridad y accesibilidad". Eroski Consumer: 4-11.

Eurobarometer

2001. "Attitudes of Europeans to Disability". Comisión Europea. Disponible en: http://europa.eu.int/ comm/public_opinion/archives/eb/ebs_149_en.pdf (Consultado Agosto 2013)

Eurostat

2005. Tourism in the Enlarged European Union. Catálogo n ${ }^{\circ}$ KS-NP-05-013-EN-N, Unión Europea.

Eurostat

2003. Employment of Disabled People in Europe in 2002. Catálogo n ${ }^{\circ}$ : KS-NK-03-026-EN-N, Unión Europea.

Figueiredo, E., Eusébio, C., y Kastenholz, E.

2012. "How diverse are tourists with disabilities? A pilot study on accessible leisure tourism experiences in Portugal".

Franco, P.

1999. "La formación de los profesionales del turismo". Ocio y equiparación de oportunidades de las terceras jornadas de la Cátedra de Ocio y Minusvalías. Coord. Manuel Cuenca Cabeza: 87-96

Fuguet, T.

2008. "Europa demanda más accesibilidad". Editur, Nª 07, Julio: 10-15.

Gerlin, A.

2005. “Access Denied”. Time Europe, Vol. 165 (15).

Gröschl, S.

2007. "An exploration of hr policies and practices affecting the integration of persons with disabilities in the hotel industry in major Canadian tourism destinations". International Journal of Hospitality Management, 26(3): 666-686.

Gröschl, S.

2012. Presumed incapable: Exploring the validity of negative judgments about persons with disabilities and their employability in hotel operations. Cornell Hospitality Quarterly.

Horgan-Jones, M. y Ringaert, L.

2004. Accessible Tourism in Manitoba. Disponible en: http://www.ttra.com/pub/uploads/AccessibleTourismInManitoba.htm (Consultado Agosto 2013)

Hosteltur

2013. "El turismo rural suspende e turismo accessible". Hosteltur 12 de Agosto de 2013.

Instituto Nacional de Estadística (INE)

1999. "Encuesta sobre Discapacidad, Deficiencia y Estado de la Salud, 1999".

Israeli, A.

2002. "A Preliminary Investigation of the Importance of Site Accessibility Factors for Disabled Tourists". Journal of Travel Research, Vol. 41 (1): 101-104

Kim, W. G., Stonesifer, H. W., y Han, J. S.

2012 "Accommodating the needs of disabled hotel guests: Implications for guests and management". International Journal of Hospitality Management, 31(4): 1311-1317.

McKercher, B., Packer, T., Yau, M. K., y Lam, P.

2003. "Travel agents as facilitators or inhibitors of travel: Perceptions of people with disabilities". Tourism Management, 24(4): 465-474.

Ministerio de Trabajo y Asuntos Sociales

2003. I Plan Nacional de Accesibilidad 2004-20012. Por un nuevo paradigma, el Diseño para Todos, hacia la plena igualdad de oportunidades.

Ministerio Federal de Economía y Tecnología de Alemania

2004. Economic Impulses of Accesible Tourism for All. Berlín.

National Disability Authority

2003. "Accessibility and Tourism". Tourism Policy Review Group. Disponible en http://www.nda.ie/ (consultado Agosto 2013).

Nyaupane, G. P., y Andereck, K. L.

2008. "Understanding travel constraints: Application and extension of a leisure constraints model". Journal of Travel Research, 46(4): 433-439. 
O’Neill, M., y Ali Knight, J.

2000. "Disability tourism dollars in western Australia hotels". FIU Hospitality Review, 18(2): 72-88.

Organización Mundial de Turismo (OMT)

2013. 'UNWTO. Tourism Highlights'. 2013 Edition UNWTO.

Ozturk, Y., Yayli, A., y Yesiltas, M.

2008. "Is the turkish tourism industry ready for a disabled customer's market?: The views of hotel and travel agency managers". Tourism Management, 29(2): 382-389.

Patterson, I., Darcy, S., y Monninghoff, M.

2012. "Attitudes and experiences of tourism operators in northern Australia toward people with disabilities". World Leisure Journal, 54(3): 215-229.

Pühretmair, F.

2004. It' "s time to make eTourism accessible”. In Miesenberger, K., Klaus, J., Zagler, W. y Burger, D. (Eds.) Computers helping people with special needs. 9th Internacional Conference, ICCHP 2004 France y Berlín.

Qualitas, A.

2004. "Conclusions of the 1st International Congress on "Tourism for all". Disponible en.: http://www. worldtourism.org/quality/E/standards2.htm (Consultado en Septiembre 2013).

Ray, N. M., y Ryder, M. E.

2003. "'Ebilities" tourism: An exploratory discussion of the travel needs and motivations of the mobility-disabled". Tourism Management, 24(1): 57-72.

Shi, L., Cole, S., y Chancellor, H. C.

2012. "Understanding leisure travel motivations of travelers with acquired mobility impairments". Tourism Management, 33(1): 228-231.

Stumbo, N.J. y Pegg, S.

2005. "Travellers and Tourists with Disabilities: A Matter of Priorities and Royalties". Tourism Review Internacional, Vol. 8, $\mathrm{N}^{\circ}$ 3: 195-209.

Tantawy, A., Kim, W. G., y Pyo, S.

2005. "Evaluation of hotels to accommodate disabled visitors". Journal of Quality Assurance in Hospitality \& Tourism, 5(1): 91 - 101.

Teorisme Vlaanderen

2001. "Tourism for all in the European Union", Status Report on Tourist Accommodation Schemes in Europe. Meeting of EU Ministers of Tourism, "Tourism for all", 2001. Disponible en: http://www. toegankelijkheidsbureau.be/docs/Tourism\%20for\%20All\%20Report\%20Final\%20SEP2001b.pdf

Turco, D. M., Stumbo, N., y Garncarz, J.

1998. "Tourism constraints - people with disabilities". Parks and Recreation Journal, 33(9): 78-84.

U.S. Department of Commerce

1997. "Current Population Report: Americanswith Disabilities: 1994-95”. Disponible en: http://census. gov.hhes/www/disable/sipp/disable9495 (consultado Agosto 2013).

Van Horn, L.

2012. "The united states: Travellers with disabilities". In D. Buhalis, S. Darcy \& I. Ambrose (Eds.), Best practice in accessible tourism: Inclusion, disability, ageing population and tourism (pp. 65-78). Bristol, UK: Channel View Publications.

Vogel, N.

2006. "Not marketing to people with disabilities? You're missing out. Most marketers are ignoring loyal consumer segment that has 'sizable spending power". Disponible en: http://springboard.com.s75811. gridserver.com/wpcontent/uploads/2009/08/AdvertisingAgeArticlePublished.pdf

Van Horn, L.

2002. "Travellers with Disabilities: Market Size and Trenes. Disponible en http://ncpedp.org/access/ isu-travel.htm (Consultado Septiembre 2013).

Yaniv, P., Arie, R., y Yael, B.

2011. "Dimensions of hotel experience of people with disabilities: An exploratory study". International Journal of Contemporary Hospitality Management, 23(5): 571-591.

Recibido:

$04 / 02 / 2013$

Reenviado:

$29 / 04 / 2013$

Aceptado:

$03 / 05 / 2013$

Sometido a evaluación por pares anónimos 